\title{
THE ROLE OF THE DISTANCE FUNCTION IN SOME SINGULAR PERTURBATION PROBLEM
}

\author{
ANGELA PISTOIA*
}

0. Introduction. This paper deals with the study of solutions to a class of nonlinear singularly perturbed problems of the form

$$
\begin{cases}-\varepsilon^{2} \Delta u+u=u^{p} & \text { in } \Omega \\ u>0 & \text { in } \Omega \\ u=0 \text { or } \frac{\partial u}{\partial \nu}=0 & \text { on } \partial \Omega\end{cases}
$$

where $\Omega$ is a bounded smooth domain of $\mathbb{R}^{N}, N \geq 2, \varepsilon>0,1<p<\frac{N+2}{N-2}$ if $N \geq 3$ or $p>1$ if $N=2$ and $\nu$ is the unit outward normal at the boundary of $\Omega$.

A solution of the Dirichlet problem can be interpretated as a steady state of the corresponding reaction-diffusion equation $u_{t}=\varepsilon^{2} \Delta u-u+u^{p}$, which arises in a numbers of problems, such as dynamic population and pattern formation theories and chemical reactor theory. The Neumann problem is known as the stationary equation of Keller-Segal system in chemotaxis. It can also be seen as the limiting stationary equation of the Gierer-Heinhardt system in biological pattern formation.

\section{Neumann problem}

In the pioneering papers [29], [31] and [32] Lin, $\mathrm{Ni}$ and Takagi established the existence of least energy solutions and showed that for $\varepsilon$ small enough the least energy solution has only one local maximum point $x_{\varepsilon}$ which belongs to $\partial \Omega$. Moreover the limit point $x_{0}=\lim _{\varepsilon \rightarrow 0} x_{\varepsilon}$ satisfies $H\left(x_{0}\right)=\max _{x \in \partial \Omega} H(x)$, where $H$ denotes the mean curvature of $x$ at $\partial \Omega$. In [33] $\mathrm{Ni}$ and Takagi constructed boundary spike solutions for axially symmetric domains. In [39] Wei studied the general domain case and proved that for single boundary spike solutions the boundary spike must approach a critical point of the mean curvature. He also proved that for any nondegenerate critical point of the mean curvature one can construct bondary spike solutions whose spike approaches such a point.

In [22] Gui constructed multiple boundary spike layer solutions at multiple local maximum points. In [44] Wei and Winter constructed multiple boundary spike layer solutions at multiple nondegenerate critical points of $H$. In [24] the authors proved that for any fixed integers $K$ there exist boundary $K$-peaks solutions at a local minimum point of $H$.

In [40] and in [41] Wei proved the existence of single interior spike solutions of (0.1) under some restricted geometric conditions on $\Omega$. In [42] and [20] the authors constructed single interior spike solutions by using the distance function $\operatorname{dist}(x, \partial \Omega)$. More precisely in [42] Wei proved that for any local maximum point $x_{0}$ of the distance function there exists a family of solutions with a single maximum point which approaches $x_{0}$. In [35]the author proved the existence of a symmetric single interior spike solution in symmetric domains, by using a degree argument.

*Dipartimento Me.Mo.Mat., via A.Scarpa 16, 00100 Roma, Italy. (pistoia@dmmm.uniroma1.it). 
In [23] Gui constructed multiple interior peak solutions. It was shown that for any fixed positive integer $K$ there exists a solution of (0.1) which has exactly $K$ maximum points $x_{\varepsilon}^{1}, \ldots, x_{\varepsilon}^{K}$ such that $\mathcal{D}_{K}\left(x_{\varepsilon}^{1}, \ldots, x_{\varepsilon}^{K}\right)$ converges to $\max \left\{\mathcal{D}_{K}\left(x^{1}, \ldots, x^{K}\right) \mid x^{i} \in\right.$ $\Omega, i=1, \ldots, K\}$ as $\varepsilon$ tends to zero, where

$$
\mathcal{D}_{K}\left(x^{1}, \ldots, x^{K}\right)=\min \left\{\operatorname{dist}\left(x^{i}, \partial \Omega\right), \frac{\left|x^{j}-x^{l}\right|}{2} \mid i, j, l=1 \ldots, K, j \neq l\right\} .
$$

Cerami and Wei in [9] and Yan in [37] some multiplicity results are obtained by using Ljusternik-Schnirelman category. In [25] Kowalczyk proved that any "nondegenerate stationary lattice" supports a multiple spike layer solution.

We would like to point out that Bates and Fusco in [5] got similar results for the Cahn-Hilliard equation. By using a "quasi-invariant" manifold approach they established the existence of a stationary solution with many nuclei and they also gave a criteria for the asymptotic location of those nuclei as $\varepsilon \rightarrow 0$ in terms of the geometry of the domain.

\section{Dirichlet problem}

Multiplicity results about the Dirichlet problem were firstly obtained by Benci, Cerami and Passaseo in [2] and [3], by using the Ljusternik-Schnirelman category. Successively $\mathrm{Ni}$ and Wei in [34] established the existence of a least energy solution. They proved that as $\varepsilon \rightarrow 0$ the least energy soluton has exactly one local maximum point and this local maximum point tends to a point which attains the global maximum of the distance function $\operatorname{dist}(x, \partial \Omega)$. In [39] Wei proved that for any local maximum $x_{0}$ of the distance function there exists a family of solutions with a single global maximum point which approaches $x_{0}$. In [16] Del Pino, Felmer and Wei proved the existence of single-peaked solutions at any "suitable" critical point of the distance function. In [28] $\mathrm{Li}$ and Nirenberg proved another result which involves the critical points of the distance function. More precisely they show that if the Brower degree $\operatorname{deg}(\nabla \operatorname{dist}(\cdot, \partial \Omega), V, 0) \neq 0$ where $V$ is a suitable subset of $\Omega$, then there exist a family of solutions with a unique local maximum point which converges to a critical point of the distance function.

In [7] and [8] Cao, Dancer, Noussair and Yan constructed $K$-peak solutions with the peaks near the local maximum points or saddle points $x_{1}, \ldots, x_{K}$ of $\operatorname{dist}(\cdots, \partial \Omega)$, provided $\operatorname{dist}\left(x_{i}, \partial \Omega\right)=\operatorname{dist}\left(x_{j}, \partial \Omega\right)$ for any $i$ and $j$. In [17] Del Pino, Felmer and Wei used a variational method to construct a $K$-peak solution with its peaks close to some local maximum points $x_{1}, \ldots, x_{K}$ of $\operatorname{dist}(\cdots, \partial \Omega)$, provided $\max _{i} \operatorname{dist}\left(x_{i}, \partial \Omega\right)$ is small when compared with the distance between $x_{1}, \ldots, x_{K}$. In [15] Dancer and Wei proved the existence of two-peak solutions. Concerning the effect of the domain topology on the existence of multipeak solution Dancer and Yan in [13] proved that if the homology of the domain is nontrivial, then for any positive integer $K$ problem (0.1) has at least one $K$-peak solution. In [14] Dancer and Yan assumed that the distance function has $K$ isolated compact connected critical sets $T_{1}, \ldots, T_{K}$ satisfying $\operatorname{dist}(x, \partial \Omega)=c_{j}=$ constant for all $x \in T_{j}, \min _{i \neq j} \mathrm{~d}\left(T_{j}, T_{i}\right)>2 \max _{1 \leq j \leq K} \mathrm{~d}\left(T_{j}, \partial \Omega\right)$ and the critical group of each critical set $T_{i}$ is nontrivial. They constructed a solution which has exactly one local maximum point in a small neighbourhood of $T_{i}$ for $i=1, \ldots, K$. Moreover they proved that if $\Omega$ is strictly convex problem $(0.1)$ does not have any $K$-peak solution. 
In this paper we describe some results obtained by Grossi, Wei and the author in [20] and in [21].

In [20] the authors proved that any critical point "topologically non trivial" $x_{0}$ of the distance function generates a family of single interior spike solutions.

THEOREM 0.1 Let $x_{0}$ be a critical point of dist $(\cdot, \partial \Omega)$. Assume $c=\operatorname{dist}\left(x_{0}, \partial \Omega\right)$ is a critical value topologically nontrivial (see Definition (2.4)). Then for $\varepsilon$ small enough there exists a family of solutions $u_{\varepsilon}$ of (0.1), whose maximum point tends to a critical point $x_{0}^{\prime}$ of the distance function with $\operatorname{dist}\left(x_{0}^{\prime}, \partial \Omega\right)=\operatorname{dist}\left(x_{0}, \partial \Omega\right)$.

Moreover they proved that the peak of any single solution must converge to a critical point of the distance function.

THEOREM 0.2. Let $u_{\varepsilon}$ be a solution of (0.1) with exactly one local interior maximum point $x_{\varepsilon}$. If $x_{0}=\lim _{\varepsilon \rightarrow 0} x_{\varepsilon} \in \Omega$ then $x_{0}$ is a critical point of $\mathrm{d}_{\partial \Omega}$.

In [21] the authors proved that any critical point "topologically non trivial" of the function $\mathcal{D}_{K}$ generates a $K$-peaks solution.

THEOREM 0.3. Let $X_{0}=\left(x_{0}^{1}, \ldots, x_{0}^{K}\right)$ be a critical point of $\mathcal{D}_{K}$. Assume $\mathcal{D}_{K}\left(X_{0}\right)>0$ is a critical value topologically nontrivial (see Definition (2.4)). Then for $\varepsilon$ small enough there exists a family of solutions $u_{\varepsilon}$ of (0.1), with Neumann boundary condition, whose $K$ maximum points $x_{\varepsilon}^{1}, \ldots, x_{\varepsilon}^{K}$ tend to a point $\hat{X}_{0}=\left(\hat{x}_{0}^{1}, \ldots, \hat{x}_{0}^{K}\right)$ such that $\mathcal{D}_{K}\left(\hat{X}_{0}\right)=\mathcal{D}_{K}\left(X_{0}\right), \hat{x}_{0}^{i} \in \Omega, \hat{x}_{0}^{i} \neq x_{0}^{j}$ for $i \neq j$ and $\hat{X}_{0}$ is a critical point of $\mathcal{D}_{K}$.

Moreover they proved that the $K$ peaks of any single solution must converge to a critical point of the function $\mathcal{D}_{K}$.

THEOREM 0.4. Let $u_{\varepsilon}$ be a solution of (0.1), with Neumann boundary condition, with exactly $K$ local interior maximum points $x_{\varepsilon}^{1}, \ldots, x_{\varepsilon}^{K}$ and let $x_{0}^{i}=\lim _{\varepsilon \rightarrow 0} x_{\varepsilon}^{i}$ for $i=1, \ldots, K$. If $x_{0}^{i} \in \Omega$ then $x_{0}^{i} \neq x_{0}^{j}$ for $i \neq j$ and $\left(x_{0}^{1}, \ldots, x_{0}^{K}\right)$ is a critical point of $\mathcal{D}_{K}$.

The method used to prove the results relies on an idea of Bahri (see [1]).

Firstly for $\varepsilon$ small enough we reduce the problem of finding a single-peak or a multi-peak solution for (0.1) to that of finding a critical point for a function $K_{\varepsilon}$ defined in a finite dimensional domain.

Secondly we compute the asymptotic expansion of the function $K_{\varepsilon}$, in order to point out the connection between $K_{\varepsilon}$ and function $\mathcal{D}_{K}$. Such an expansion allows us to prove that any "topologically nontrivial" critical point of the function $\mathcal{D}_{K}$ generates a $K$-peak solution.

Finally we compute the asymptotic expansion of the function $\nabla K_{\varepsilon}$, in order to point out the connection between $\nabla K_{\varepsilon}$ and $\nabla \mathcal{D}_{K}$. Such an expansion allows us to prove that the $K$ peaks of any single solution must converge to a critical point of the function $\mathcal{D}_{K}$.

We would like to emphasize that $\mathcal{D}_{K}$ is a Lipschitz continuous function which may be not smooth. So a suitable notion of critical points for non-smooth functions is needed. The generalized gradient introduced by Clarke (see [11]) becomes our main tool. The new idea in [20] and in [21] is to evaluate the gradient of $K_{\varepsilon}$ in terms of the generalized gradient of Clarke of the function $\mathcal{D}_{K}$. By this result, we were able to get some new results and also to clarify many results that were previously known. 
The paper is organized as follows. In Section 1 we recall some properties of the generalized gradient of Clarke. In Section 2 we introduce the notion of "topologically nontrivial" critical values for locally Lipschitz continuous function. In Section 3 we study the distance function and the function $\mathcal{D}_{K}$ and we give a criteria to localize critical points of $\mathcal{D}_{K}$. In Section 4 we recall some results obtained by $\mathrm{Ni}$ and Wei in [34]. In Section 5 we study the one-peak solutions. In Section 6 we study the multi-peak solutions. In Section 7 we give some examples.

1. The generalized gradient. Let $D$ be a smooth bounded domain of $\mathbb{R}^{N}$. Let $f: D \longrightarrow \mathbb{R}$ be a Lipschitz continuous function. We recall the following definition due to Clarke (see [11]).

DEFINITION 1.1. The generalized gradient of $f$ at $x \in D$ is the set:

$$
\partial f(x)=\left\{\alpha \in \mathbb{R}^{N} \mid f^{o}(x, v) \geq \alpha \cdot v \forall v \in \mathbb{R}^{N}\right\}
$$

where the generalized directional derivative $f^{o}(x, v)$ is defined by

$$
f^{o}(x ; v)=\limsup _{\substack{h \rightarrow 0+\\ \lambda \rightarrow 0^{+}}} \frac{f(x+h+\lambda v)-f(x+h)}{\lambda} .
$$

If $f$ is continuously differentiable at $x$ then $\partial f(x)=\{\nabla f(x)\}$. If $f$ is only differentiable at $x, \partial f(x)$ can contain points other than $\nabla f(x)$. For example, if $f(x)=x^{2} \sin \frac{1}{x}$ then it is easy to show that $f^{o}(0 ; v)=|v|$ and so $\partial f(0)=[-1,1]$, which contains the derivative $f^{\prime}(0)=0$.

DEFINITION 1.2. The function $f$ is said to be regular at $x \in D$ provided that for any $v \in \mathbb{R}^{N}$ there exists the usual one-sided directional derivative $f^{\prime}(x ; v)=$ $\lim _{t \rightarrow 0^{+}} \frac{f(x+t v)-f(x)}{t}$ and $f^{\prime}(x ; v)=f^{o}(x ; v)$.

By ([11], Proposition 2.2.4) and ([11], (b) of Proposition 2.3.6) we deduce

Proposition 1.3. If $\partial f(x)$ reduces to a singleton $\{\alpha\}$ then $f$ is differentiable at $x$ and $\nabla f(x)=\alpha$. Conversely, if $f$ is differentiable and regular at $x$ then $\partial f(x)=$ $\{\nabla f(x)\}$.

It is useful to point out the following property (see [11], Proposition 2.1.5).

REMARK 1.4. Let $x_{n}$ and $\alpha_{n}$ be sequences in $\mathbb{R}^{N}$ such that $x_{n} \in D$ and $\alpha_{n} \in$ $\partial f\left(x_{n}\right)$. Suppose that $x_{n}$ converges to $x$ and $\alpha_{n}$ converges to $\alpha$. Then $\alpha \in \partial f(x)$.

Now let us suppose $x=\left(x_{1}, x_{2}\right)$. We denote by $\partial_{1} f\left(x_{1}, x_{2}\right)$ the (partial) generalized gradient of $f\left(\cdot, x_{2}\right)$ at $x_{1}$ and by $\partial_{2} f\left(x_{1}, x_{2}\right)$ that of $f\left(x_{1}, \cdot\right)$ at $x_{2}$. The following result holds (see [11], Proposition 2.3.15).

REMARK 1.5. If $f$ is regular at $\left(x_{1}, x_{2}\right)$ then

$$
\partial f\left(x_{1}, x_{2}\right) \subset \partial_{1} f\left(x_{1}, x_{2}\right) \times \partial_{2} f\left(x_{1}, x_{2}\right) .
$$

Let us recall another useful result. Assume that $\left\{f_{i}\right\}_{i \in \mathcal{I}}$ is a finite collection of Lipschitz continuous functions defined on $D$. The function

$$
f(x)=\min \left\{f_{i}(x) \mid i \in \mathcal{I}\right\}
$$


is easily seen to be a Lipschitz continuous function. For any $x \in D$ we let $\mathcal{I}(x)$ denote the set of indices $i$ for which $f(x)=f_{i}(x)$ (i.e. the indices at which the minimum defining $f$ is attained). Then the following result holds (see [11], Proposition (2.3.12)). and

Proposition 1.6. If $f_{i}$ is regular at $x$ for any $i \in \mathcal{I}(x)$ then $f$ is regular at $x$

$$
\partial f(x)=\operatorname{co}\left\{\partial f_{i}(x) \mid i \in \mathcal{I}(x)\right\}
$$

Finally we give the definition of a critical point for a nonsmooth function.

Definition 1.7. A point $x_{0}$ in $D$ is said to be a critical point of $f$ if $0 \in \partial f\left(x_{0}\right)$. $A$ real number $c$ is said to be a critical value of $f$ if there exists a critical point $x_{0}$ of $f$ such that $f\left(x_{0}\right)=c$.

By Definition (1.1) we easily deduce that if $x_{0}$ is a minimum point or a maximum point for a Lipschitz continuous function $f$ then $0 \in \partial f\left(x_{0}\right)$.

2. Critical values topologically nontrivial. In this section we recall a result of the critical point theory. The following one is given by Ramos in [36] and it is a jointed version of the classical linking theorem and the local saddle point proved in [30]. Although it concerns $C^{1}$-function, it is possible to extend such a result to Lipschitz continuous function, by using deformation lemma proved by Chang in [10].

We consider three compact subsets $\partial Q, Q$ and $A$ of $D$ such that

$$
\partial Q \subset Q \text { and } Q \cap A=\emptyset .
$$

$\partial Q$ is not necessarily the topological boundary of $Q$ and $A$ can be the empty set.

We define the class:

$$
\Gamma=\left\{\gamma \in C^{0}([0,1] \times Q, D \backslash A) \mid \gamma_{0} \equiv I d, \gamma_{t_{\partial Q}} \equiv I d \forall t \in[0,1]\right\},
$$

where $I d$ is the identity map. We note that $\Gamma \neq \emptyset$ because $I d \in \Gamma$.

Definition 2.1. Let $S$ be a subset of $D$. We say that $S$ links $Q$ via $\partial Q$ by homotopy in $D \backslash A$ if

$$
S \cap \partial Q=\emptyset \quad \text { and } \quad \gamma_{1}(Q) \cap S \neq \emptyset \forall \gamma \in \Gamma .
$$

It is useful to point out the following fact.

Remark 2.2. Assume $\partial Q_{1}, Q_{1}, A_{1}$ and $S_{1}$ and $\partial Q_{2}, Q_{2}, A_{2}$ and $S_{2}$ are two families of subset of $D$ which satisfy (2.1) and (2.2). Then $\partial Q=\left(\partial Q_{1} \times Q_{2}\right) \cup\left(Q_{1} \times\right.$ $\left.\partial Q_{2}\right), Q=Q_{1} \times Q_{2}, A=\left(A_{1} \times S_{2}\right) \cup\left(S_{1} \times A_{2}\right)$ and $S=S_{1} \times S_{2}$ are subsets of $D \times D$ which satisfy (2.1) and (2.2).

The following result holds.

Theorem 2.3. Let $f: D \longrightarrow \mathbb{R}$ be a Lipschitz continuous function. Assume $S$ links $Q$ via $\partial Q$ by homotopy in $D \backslash A$ and

$$
\max _{\partial Q} f<\min _{S} f \leq \max _{Q} f<\min _{A} f .
$$

Let

$$
c=\inf _{\gamma \in \Gamma} \max _{u \in Q} f\left(\gamma_{1}(u)\right) .
$$


If $c \in \mathbb{R}$ and the set $\{x \in D$ s.t. $c-\varepsilon \leq f(x) \leq c+\varepsilon\}$ is complete for some $\varepsilon>0$ then $c$ is a critical value of $f$.

If $A=\emptyset$ we get the classical linking theorem. The "local saddle point" of [30] is a particular case of the previous theorem when $A \neq \emptyset$.

In the following definition we introduce the notion of critical values of a Lipschitz continuous function $f: D \longrightarrow \mathbb{R}$ which are "stable" with respect to suitable perturbations (see [20], Definition (1.7) and [21], Definition (1.11))

DEFINITION 2.4. We say that $c$ is a critical value topologically nontrivial of $f$ if there exists a family of subsets $\partial Q_{\delta}, Q_{\delta}, A_{\delta}$ and $S_{\delta}$ of $D$ which satisfy (2.1), (2.2) and (2.3), with the properties

$$
\max _{\partial Q_{\delta}} f<\min _{S_{\delta}} f \leq c \leq \max _{Q_{\delta}} f<\min _{A_{\delta}} f
$$

and

$$
\lim _{\delta \rightarrow 0} \min _{S_{\delta}} f=\lim _{\delta \rightarrow 0} \max _{Q_{\delta}}=c .
$$

We point out that if we assume that the sets $\left\{x \in D\right.$ s.t. $\left.c^{\prime}-\varepsilon \leq f(x) \leq c^{\prime}+\varepsilon\right\}$ are complete for any $c^{\prime}$ close enough to $c$ and for some $\varepsilon>0$ then by Theorem (2.3) we deduce that $c$ is a critical value of $f$.

3. The distance function and the function $\mathcal{D}_{K}$.. Let $\Omega$ be a smooth open bounded domain of $\mathbb{R}^{N}$.

Definition 3.1. Let $\mathrm{d}_{\partial \Omega}: \Omega \longrightarrow \mathbb{R}$ be the distance function defined by $\mathrm{d}_{\partial \Omega}(x)=$ $\operatorname{dist}(x, \partial \Omega)=\min _{y \in \partial \Omega}|x-y|$.

It is well known that $\mathrm{d}_{\partial \Omega}$ is a Lipschitz continuous function. By using (see [11], Corollary 2, p. 87) we can compute the generalized gradient of the distance function.

REMARK 3.2. For any $x \in \Omega$ we have

$$
\begin{aligned}
\partial \mathrm{d}_{\partial \Omega}(x)= & \left\{\int_{\partial \Omega} \nu^{(i)}(y) d \mu_{x}(y) \mid\right. \\
& d \mu_{x}(y) \text { is a bounded Borel measure on } \partial \Omega, \\
& \left.\int_{\partial \Omega} d \mu_{x}(y)=1, \operatorname{supp}\left(d \mu_{x}(y)\right) \subset \Pi_{\partial \Omega}(x)\right\},
\end{aligned}
$$

where

$$
\Pi_{\partial \Omega}(x)=\left\{y \in \partial \Omega|| y-x \mid=\mathrm{d}_{\partial \Omega}(x)\right\}
$$

and $\nu^{(i)}(y)$ denotes the unit inward normal at the point $y$ of $\partial \Omega$.

By ([11], Corollary 2, p. 87) we deduce that the distance function is regular at any $x \in \Omega$. Therefore by Proposition (1.3) we get

REMARK 3.3. $\mathrm{d}_{\partial \Omega}$ is differentiable at $x$ if and only if $\Pi_{\partial \Omega}(x)$ reduces to a singleton $\{\pi(x)\}$ and $\nabla \mathrm{d}_{\partial \Omega}(x)=\nu^{(i)}(\pi(x))$, where $\nu^{(i)}(\pi(x))$ denotes the unit inward normal at $\pi(x)$. 
Finally since $\Omega$ is smooth we have the following property.

Proposition 3.4. There exists a neighbourhood $\mathcal{U}$ of the boundary of $\Omega$ such that $0 \notin \partial \mathrm{d}_{\partial \Omega}(x)$ for any $x \in \mathcal{U} \cap \Omega$.

Now let us introduce the function $\mathcal{D}_{K}$ which will play a crucial role in the next sections.

Definition 3.5. Let $K \geq 1$ be an integer. Set $\Omega^{K}=\Omega \times \ldots \times \Omega$. Let $\mathcal{D}_{K}$ : $\Omega^{K} \longrightarrow \mathbb{R}$ be defined by

$$
\mathcal{D}_{K}(X)=\min \left\{\mathrm{d}_{\partial \Omega}\left(x^{i}\right), \frac{\left|x^{j}-x^{l}\right|}{2} \mid i, j, l=1 \ldots, K, j \neq l\right\} .
$$

Let us point out that

$$
\mathcal{D}_{1}(x)=\mathrm{d}_{\partial \Omega}(x) \quad \forall x \in \Omega .
$$

Set

$$
\mathcal{M}_{K}(\Omega)=\left\{X=\left(x^{1}, \ldots, x^{K}\right) \in \Omega^{K} \mid x^{i} \neq x^{j}, i \neq j, i, j=1, \ldots, K\right\} .
$$

By using the regularity of the distance function and Proposition (1.6) we can compute the generalized gradient of $\mathcal{D}_{K}$.

Lemma 3.6. For any $X \in \mathcal{M}_{K}(\Omega)$ we have that $\beta(X) \in \partial \mathcal{D}_{K}(X)$ if and only if

$$
\beta(X)=\left(a_{1} \alpha\left(x^{1}\right)+\frac{1}{2} \sum_{\substack{j=1 \\ j \neq 1}}^{K} b_{1 j} \frac{x^{1}-x^{j}}{\left|x^{1}-x^{j}\right|}, \ldots, a_{K} \alpha\left(x^{K}\right)+\frac{1}{2} \sum_{\substack{j=1 \\ j \neq K}}^{K} b_{1 j} \frac{x^{K}-x^{j}}{\left|x^{K}-x^{j}\right|}\right),
$$

with $\alpha\left(x^{i}\right) \in \partial \mathrm{d}_{\partial \Omega}\left(x^{i}\right), a_{i}, b_{j l} \geq 0, b_{j l}=b_{l j}, \sum_{i=1}^{K} a_{i}+\frac{1}{2} \sum_{\substack{j, l=1 \\ l \neq j}}^{K} b_{j l}=1$.

In particular by Lemma (3.6) we deduce that if $x^{1}, \ldots, x^{K}$ are $K$ different critical points of the distance function then $X=\left(x^{1}, \ldots, x^{K}\right)$ is a critical point of $\mathcal{D}_{K}$.

Next results generalizes Proposition (3.4). More precisely we prove that there is not any critical point of $\mathcal{D}_{K}$ close to the boundary of $\mathcal{M}_{K}(\Omega)$.

Proposition 3.7. There exists a neighbourhood $\mathcal{U}$ of the boundary of $\mathcal{M}_{K}(\Omega)$ such that $0 \notin \partial \mathcal{D}_{K}(X)$ for any $X \in \mathcal{U} \cap \mathcal{M}_{K}(\Omega)$.

Proof. We prove that if $X_{\varepsilon}$ is a sequence in $\mathcal{M}_{K}(\Omega)$ such that $\lim _{\varepsilon \rightarrow 0} X_{\varepsilon}=X_{0}$ and $X_{0} \in \partial \mathcal{M}_{K}(\Omega)$, then there exists $\varepsilon_{0}>0$ and $C>0$ such that for any $\varepsilon \in\left(0, \varepsilon_{0}\right)$

$$
\left|\beta_{\varepsilon}\left(X_{\varepsilon}\right)\right| \geq C>0 \quad \forall \beta_{\varepsilon}\left(X_{\varepsilon}\right) \in \partial \mathcal{D}_{K}\left(X_{\varepsilon}\right) .
$$

We proceed by induction on the number $K$.

Let $K=1$ and let $x_{\varepsilon}$ be a sequence in $\Omega$ such that $x_{0}=\lim _{\varepsilon} x_{\varepsilon} \in \partial \Omega$. By Remark (3.3) and Remark (3.4) it follows that for $\varepsilon$ small enough $\partial \mathcal{D}_{1}\left(x_{\varepsilon}\right)=\left\{\nu^{(i)}\left(\pi\left(x_{\varepsilon}\right)\right)\right\}$ and the claim follows.

Suppose the claim to be true for any integer $1 \leq H \leq K-1$. Let us prove the claim for $K$. 
Let $X_{\varepsilon}$ be a sequence in $\mathcal{M}_{K}(\Omega)$ such that $\lim _{\varepsilon \rightarrow 0} X_{\varepsilon}=X_{0}$ and $X_{0} \in \partial \mathcal{M}_{K}(\Omega)$. Then we have either

(i) $\exists i, j \in\{1, \ldots, K\}$ s.t. $x_{0}^{i} \neq x_{0}^{j}$,

or

(ii) $x_{0}^{1}=\ldots=x_{0}^{K} \in \partial \Omega$,

or

(iii) $x_{0}^{1}=\ldots=x_{0}^{K} \in \Omega$.

By using Lemma (3.6) and inductive assumptions the claim easily follows.

Next results allows us to localize some special critical points of the function $\mathcal{D}_{K}$.

Proposition 3.8. Let $\left(x^{1}, \ldots, x^{K}\right) \in \mathcal{M}_{K}(\Omega)$ be a critical point of $\mathcal{D}_{K}$. Assume that for any integer $1 \leq H \leq K-1$ and for any set of indices $\left\{i_{1}, \ldots, i_{H}\right\} \subset\{1, \ldots, K\}$ $\left(x^{i_{1}}, \ldots, x^{i_{H}}\right)$ is not a critical point of $\mathcal{D}_{H}$. Then $\mathrm{d}_{\partial \Omega}\left(x^{i}\right)=\frac{\left|x^{l}-x^{h}\right|}{2}$ for any $i, l, h$ and $0 \in \operatorname{co}\left\{\alpha\left(x^{i}\right) \mid \alpha\left(x^{i}\right) \in \partial \mathrm{d}_{\partial \Omega}\left(x^{i}\right), i=1, \ldots, K\right\}$.

Proof. We argue by contradiction. Then we have either

(i) $\exists i, j \in\{1, \ldots, K\}$ s.t. $\mathcal{D}_{K}(X)<\frac{\left|x^{i}-x^{j}\right|}{2}$,

or

(ii) $\forall l, h \in\{1, \ldots, K\} \quad \mathcal{D}_{K}(X)=\frac{\left|x^{l}-x^{h}\right|}{2}$ and $\exists i \in\{1, \ldots, K\}$ s.t.

$\mathcal{D}_{K}(X)<\mathrm{d}_{\partial \Omega}\left(x^{i}\right)$.

By using Lemma (3.6) a contradiction arises in both cases.

In particular by Proposition (3.8) and by Remark (3.3) we deduce the following characterization of the critical points of $\mathcal{D}_{2}$.

COROLlary 3.9. Let $\left(x^{1}, x^{2}\right) \in \mathcal{M}_{2}(\Omega)$ be a critical point of $\mathcal{D}_{2}$ such that the distance function is differentiable at $x^{1}$ and $x^{2}$. Then $\mathrm{d}_{\partial \Omega}\left(x^{1}\right)=\mathrm{d}_{\partial \Omega}\left(x^{2}\right)=\frac{\left|x^{1}-x^{2}\right|}{2}$ and $\nu^{(i)}\left(\pi\left(x^{1}\right)\right)=-\nu^{(i)}\left(\pi\left(x^{2}\right)\right)=\frac{x^{2}-x^{1}}{\left|x^{2}-x^{1}\right|}$.

4. Some preliminary results. Let us introduce the ground state solution $U$. We recall the following results (see, for example, [6], [19] and [26]).

THEOREM 4.1. The equation:

$$
\begin{cases}-\Delta u+u=u^{p} & \text { in } \mathbb{R}^{N} \\ u(x) \rightarrow 0 & \text { for }|x| \rightarrow+\infty\end{cases}
$$

possesses a unique non trivial regular solution $U$ with the following properties:

(i) $U(x)>0 \quad \forall x \in \mathbb{R}^{N}$

(ii) $U$ is spherically symmetric, i.e. $U(x)=U(r)$ where $r=|x|$, and $U$ decreases with respect to $r$,

(iii) $U \in \mathcal{C}^{2}\left(\mathbb{R}^{N}\right)$,

(iv) $U$ together with its derivatives up to order 2 have exponential decay at infinity, that is there exist $C>0$ and $\delta>0$ such that $\left|D^{\alpha} U(x)\right| \leq C e^{-\delta|x|} \forall x \in \mathbb{R}^{N}$ and $|\alpha| \leq 2$.

(v) there exists $\beta>0$ such that $\lim _{r \rightarrow \infty} r^{\frac{n-1}{2}} e^{r} U(r)=\beta>0$.

Let us introduce some notation. Set $\Omega_{\varepsilon}=\{y \mid \varepsilon y \in \Omega\}$ and for $x \in \Omega \Omega_{\varepsilon, x}=$ $\{y \mid \varepsilon y+x \in \Omega\}$. Of course solving problem (0.1) is equivalent to solve the rescaled 
problem

$$
\begin{cases}-\Delta u+u=u^{p} & \text { in } \Omega_{\varepsilon} \\ u=0 \text { or } \frac{\partial u}{\partial \nu}=0 & \text { in } \partial \Omega_{\varepsilon} .\end{cases}
$$

We set $\mathcal{P}_{\Omega_{\varepsilon, x}} U$ to be the unique solution of the problem

$$
\begin{cases}-\Delta u+u=U^{p} & \text { in } \Omega_{\varepsilon, x} \\ u=0 \text { or } \frac{\partial u}{\partial \nu}=0 & \text { in } \partial \Omega_{\varepsilon, x} .\end{cases}
$$

$\mathcal{P}_{\Omega_{\varepsilon, x}} U$ is the projection of the ground state $U$ into $\mathrm{H}_{0}^{1}\left(\Omega_{\varepsilon, x}\right)$ in the Dirichlet case or into $\mathrm{H}^{1}\left(\Omega_{\varepsilon, x}\right)$ in the Neumann case. The idea of projections has been introduced in [1].

Set

$$
\varphi_{\varepsilon, x}(z)=U(y)-\mathcal{P}_{\Omega_{\varepsilon, x}} U(y) \quad \text { with } \quad z=\varepsilon y+x, x \in \Omega, z \in \Omega
$$

The following estimate plays a fondamental role (see [41], Section 2 and [34], Section 4).

Lemma 4.2. For $x \in \Omega$ set

$$
\psi_{\varepsilon}(x)=-\varepsilon \log \left(\varphi_{\varepsilon, x}(x)\right) \quad \text { in the Dirichlet case }
$$

or

$$
\psi_{\varepsilon}(x)=-\varepsilon \log \left(-\varphi_{\varepsilon, x}(x)\right) \quad \text { in the Neumann case, }
$$

Then

$$
\lim _{\varepsilon \rightarrow 0} \psi_{\varepsilon}(x)=2 \mathrm{~d}_{\partial \Omega}(x) \quad \text { uniformly in } \Omega
$$

By Lemma (4.2) and by $(v)$ of Theorem (4.1) we easily deduce that

Lemma 4.3. Let for $X \in \mathcal{M}_{K}(\Omega)$

$$
\Phi_{\varepsilon}(X)=-\varepsilon \log \left[-\sum_{i=1}^{K} \varphi_{\varepsilon, x^{i}}\left(x^{i}\right)+\sum_{\substack{j, l=1 \\ j \neq l}}^{K} U\left(\frac{\left|x^{j}-x^{l}\right|}{\varepsilon}\right)\right] .
$$

Then in the Neumann case

$$
\lim _{\varepsilon \rightarrow 0} \Phi_{\varepsilon}(X)=2 \mathcal{D}_{K}(X) \quad \text { uniformly in } \mathcal{M}_{K}(\Omega)
$$


5. Existence of one-peak solutions. Let $\mathrm{H}_{\varepsilon}$ be the Hilbert space

$$
\mathrm{H}_{\varepsilon}=\mathrm{H}^{2}\left(\Omega_{\varepsilon}\right) \cap \mathrm{H}_{0}^{1}\left(\Omega_{\varepsilon}\right) \text { in the Dirichlet case }
$$

or

$$
\mathrm{H}_{\varepsilon}=\left\{u \in \mathrm{H}^{2}\left(\Omega_{\varepsilon}\right) \mid \frac{\partial u}{\partial \nu_{\varepsilon}}=0 \text { on } \partial \Omega_{\varepsilon}\right\} \quad \text { in the Neumann case }
$$

Define

$$
\mathcal{S}_{\varepsilon}(u)=\Delta u-u+\left(u^{+}\right)^{p} \quad \text { for } \quad u \in \mathrm{H}_{\varepsilon} .
$$

Then solving equation (0.1) or equation (4.2) is equivalent to solve the following one

$$
\mathcal{S}_{\varepsilon}(u)=0, \quad u \in \mathrm{H}_{\varepsilon} .
$$

Let us consider the linearized operator $\mathcal{L}_{\varepsilon}: \mathrm{H}_{\varepsilon} \longrightarrow \mathrm{L}^{2}\left(\Omega_{\varepsilon}\right)$ given by

$$
\mathcal{L}_{\varepsilon}(v)=\Delta v-v+p \mathcal{P}_{\Omega_{\varepsilon, x}} U^{p-1} v .
$$

It is easy to see that the cokernel of $\mathcal{L}_{\varepsilon}$ coincides with its kernel. Choose approximate cokernel and kernel as

$$
\begin{aligned}
& \mathcal{K}_{\varepsilon, x}=\operatorname{span}\left\{\frac{\partial \mathcal{P}_{\Omega_{\varepsilon, x}} U}{\partial x_{i}} \mid i=1, \ldots, N\right\} \subset \mathrm{H}^{2}\left(\Omega_{\varepsilon}\right), \\
& \mathcal{C}_{\varepsilon, x}=\operatorname{span}\left\{\frac{\partial \mathcal{P}_{\Omega_{\varepsilon, x}} U}{\partial x_{i}} \mid i=1, \ldots, N\right\} \subset \mathrm{L}^{2}\left(\Omega_{\varepsilon}\right) .
\end{aligned}
$$

Now we state the following lemmas, which allow us to reduce problem (4.2) to a finite dimensional problem.

LEMMA 5.1. For any compact set $K \subset \Omega$ there exists $\varepsilon_{0}>0$ such that for any $\varepsilon \in\left(0, \varepsilon_{0}\right)$ and $x \in K$ there exists a unique $\Phi_{\varepsilon, x} \in \mathcal{K}_{\varepsilon, x}^{\perp}$ such that

$$
\mathcal{S}_{\varepsilon}\left(\mathcal{P}_{\Omega_{\varepsilon, x}} U+\Phi_{\varepsilon, x}\right) \in \mathcal{C}_{\varepsilon, x}
$$

Moreover $\Phi_{\varepsilon, x}$ is $C^{1}$ in $x$ and

$$
\left\|\Phi_{\varepsilon, x}\right\|_{\mathrm{H}^{2}\left(\Omega_{\varepsilon}\right)} \leq C e^{-(1+\sigma) \frac{\mathrm{d} \partial \Omega}{\varepsilon}}
$$

where $C$ is a positive constant and $\sigma=\min \{1, p-1\}$.

Proof. The proof relies on a contraction mapping argument. The claim can be proved by collecting some results obtained in [41] and [42].

Now we define the function $K_{\varepsilon}: \Omega \longrightarrow \mathbb{R}$

$$
K_{\varepsilon}(x)=J_{\varepsilon}\left(\mathcal{P}_{\Omega_{\varepsilon, x}} U+\Phi_{\varepsilon, x}\right),
$$

where the "rescaled" energy functional $J_{\varepsilon}: \mathrm{H}^{1}\left(\Omega_{\varepsilon}\right) \longrightarrow \mathbb{R}$ is defined by

$$
J_{\varepsilon}(u)=\left[\frac{1}{2} \int_{\Omega_{\varepsilon}}\left(|\nabla u|^{2}+u^{2}\right)-\frac{1}{p+1} \int_{\Omega_{\varepsilon}}\left(u^{+}\right)^{p+1}\right] .
$$


Now we evaluate the asymptotic expansion of $K_{\varepsilon}$.

Proposition 5.2. $x_{\varepsilon}$ is a critical point of $K_{\varepsilon}$ if and only if $u_{\varepsilon}=\mathcal{P}_{\Omega_{\varepsilon, x_{\varepsilon}}} U+\Phi_{\varepsilon, x_{\varepsilon}}$ is a solution of 4.2. Moreover the following estimates hold uniformly on compact sets of $\Omega$

$$
K_{\varepsilon}(x)=A+\frac{1}{2} \gamma e^{-\frac{\psi_{\varepsilon}(x)}{\varepsilon}}+o\left(e^{-\frac{\psi_{\varepsilon}(x)}{\varepsilon}}\right) \text { in the Dirichlet case }
$$

or

$$
K_{\varepsilon}(x)=A-\frac{1}{2} \gamma e^{-\frac{\psi_{\varepsilon}(x)}{\varepsilon}}+o\left(e^{-\frac{\psi_{\varepsilon}(x)}{\varepsilon}}\right) \quad \text { in the Neumann case }
$$

where

$$
A=\frac{1}{2} \int_{\mathbb{R}^{N}}\left(|\nabla U|^{2}+U^{2}\right)-\frac{1}{p+1} \int_{\mathbb{R}^{N}} U^{p+1}, \gamma=\int_{\mathbb{R}^{N}} U^{p}(y) e^{-y_{1}} d y
$$

Proof. See [20], [23], [41] and [42].

The next results play a crucial role in connecting the topological structure of the sublevels of the distance function with the topological structure of the sublevels of the function $K_{\varepsilon}$.

LEMMA 5.3. Let $x_{1}^{\varepsilon}, x_{2}^{\varepsilon}$ be sequences in $\Omega$ be such that $\lim _{\varepsilon \rightarrow 0} x_{1}^{\varepsilon}=x_{1} \in \Omega, \lim _{\varepsilon \rightarrow 0} x_{2}^{\varepsilon}=$ $x_{2} \in \Omega$ and $\mathrm{d}_{\partial \Omega}\left(x_{1}\right)<\mathrm{d}_{\partial \Omega}\left(x_{2}\right)$. Then there exists $\varepsilon_{0}>0$ such that for any $\varepsilon \in\left(0, \varepsilon_{0}\right)$

$$
K_{\varepsilon}\left(x_{1}^{\varepsilon}\right)>K_{\varepsilon}\left(x_{2}^{\varepsilon}\right) \text { in the Dirichlet case }
$$

or

$$
K_{\varepsilon}\left(x_{1}^{\varepsilon}\right)<K_{\varepsilon}\left(x_{2}^{\varepsilon}\right) \text { in the Neumann case. }
$$

Proof. We prove (5.7). The proof of (5.6) is the same. By the expansion of $K_{\varepsilon}$ given in (5.5) of Proposition (5.2) we have

$$
\begin{aligned}
& K_{\varepsilon}\left(x_{2}^{\varepsilon}\right)-K_{\varepsilon}\left(x_{1}^{\varepsilon}\right)=\frac{1}{2} \gamma\left(e^{-\frac{\psi_{\varepsilon}\left(x_{1}^{\varepsilon}\right)}{\varepsilon}}-e^{-\frac{\psi_{\varepsilon}\left(x_{2}^{\varepsilon}\right)}{\varepsilon}}\right) \\
& +o\left(e^{-\frac{\psi_{\varepsilon}\left(x_{1}^{\varepsilon}\right)}{\varepsilon}}\right)+o\left(e^{-\frac{\psi_{\varepsilon}\left(x_{2}^{\varepsilon}\right)}{\varepsilon}}\right) .
\end{aligned}
$$

Since $\mathrm{d}_{\partial \Omega}\left(x_{1}\right)<\mathrm{d}_{\partial \Omega}\left(x_{2}\right)$, by Lemma (4.2) we deduce that for $\varepsilon$ small enough $\psi_{\varepsilon}\left(x_{1}^{\varepsilon}\right)<$ $\psi_{\varepsilon}\left(x_{2}^{\varepsilon}\right)$. Then by (5.8) we get

$$
e^{\frac{\psi_{\varepsilon}\left(x_{1}^{\varepsilon}\right)}{\varepsilon}}\left[K_{\varepsilon}\left(x_{2}^{\varepsilon}\right)-K_{\varepsilon}\left(x_{1}^{\varepsilon}\right)\right]=\frac{1}{2} \gamma\left[1-e^{-\frac{\psi_{\varepsilon}\left(x_{2}^{\varepsilon}\right)-\psi_{\varepsilon}\left(x_{1}^{\varepsilon}\right)}{\epsilon}}\right]+o(1)
$$

and the claim follows.

LEMMA 5.4. Let $C_{1}, C_{2}$ be two compact subsets of $\Omega$. If

$$
\min _{x \in C_{1}} \mathrm{~d}_{\partial \Omega}(x)>\max _{x \in C_{2}} \mathrm{~d}_{\partial \Omega}(x)
$$


then there exists $\varepsilon_{0}>0$ such that for any $\varepsilon \in\left(0, \varepsilon_{0}\right)$

$$
\min _{x \in C_{1}}\left(-K_{\varepsilon}\right)(x)>\max _{x \in C_{2}}\left(-K_{\varepsilon}\right)(x) \text { in the Dirichlet case, }
$$

or

$$
\min _{x \in C_{1}} K_{\varepsilon}(x)>\max _{x \in C_{2}} K_{\varepsilon}(x) \text { in the Neumann case. }
$$

Now we prove that a suitable critical point of the distance function generates a critical point of $K_{\varepsilon}$.

THEOREM 5.5. Let $c$ be a critical value topologically nontrivial of the distance function (see Definition (2.4)). Then there exists a sequence $\left(x_{\varepsilon}\right)$ of critical points of $K_{\varepsilon}$ such that $\lim _{\varepsilon \rightarrow 0} x_{\varepsilon}=x_{0}$ and $\mathrm{d}_{\partial \Omega}\left(x_{0}\right)=c$.

Proof. We prove the claim in Neumann case. In the Dirichlet case we consider the function $-K_{\varepsilon}$ and we argue in the same way. By Definition (2.4) there exist a family of subsets $\partial Q_{\delta}, Q_{\delta}, A_{\delta}$ and $S_{\delta}$ of $\Omega$ which satisfy (2.1), (2.2), (2.5) and (2.6), that is:

$$
\max _{x \in \partial Q_{\delta}} \mathrm{d}_{\partial \Omega}(x)<\min _{x \in S_{\delta}} \mathrm{d}_{\partial \Omega}(x) \leq c \leq \max _{x \in Q_{\delta}} \mathrm{d}_{\partial \Omega}(x)<\min _{x \in A_{\delta}} \mathrm{d} \partial \Omega(x) .
$$

Then by (5.9) of Lemma (5.4) there exists $\varepsilon_{0}>0$ such that for any $\varepsilon \in\left(0, \varepsilon_{0}\right)$

$$
a_{\varepsilon, \delta}=\max _{x \in \partial Q_{\delta}} K_{\varepsilon}(x)<\min _{x \in S_{\delta}} K_{\varepsilon}(x) \leq \max _{x \in Q_{\delta}} K_{\varepsilon}(x)<\min _{x \in A_{\delta}} K_{\varepsilon}(x)=b_{\varepsilon, \delta} .
$$

It is not difficult to prove that for $\varepsilon$ and $\delta$ small enough the set $\left\{x \in \Omega \mid a_{\varepsilon, \delta} \leq\right.$ $\left.K_{\varepsilon}(x) \leq b_{\varepsilon, \delta}\right\}$ is complete. Now by (5.12) and Theorem (2.3) there exists $x_{\varepsilon, \delta}$ critical point of $K_{\varepsilon}$ in $\Omega$ such that:

$$
\min _{x \in S_{\delta}} K_{\varepsilon}(x) \leq K_{\varepsilon}\left(x_{\varepsilon, \delta}\right) \leq \max _{x \in Q_{\delta}} K_{\varepsilon}(x) .
$$

Up to a subsequence we can assume that $x_{\varepsilon, \delta}$ goes to $x_{0}$ as $\varepsilon$ and $\delta$ go to 0 . It is easy to show that $\mathrm{d}_{\partial \Omega}\left(x_{0}\right)=c>0$. Therefore the claim is proved.

Finally we want to show that a family of critical points of $K_{\varepsilon}$ converges to a critical point of the distance function. Firstly we have to compute the asymptotic expansion of the gradient of $K_{\varepsilon}$.

Proposition 5.6. Let $x_{\varepsilon}$ be a sequence in $\Omega$ such that $\lim _{\varepsilon \rightarrow 0} x_{\varepsilon}=x_{0} \in \Omega$. Then

$(5.14) \nabla K_{\varepsilon}\left(x_{\varepsilon}\right)=-\frac{1}{\varepsilon} \gamma \alpha\left(x_{0}\right) e^{-\frac{\psi_{\varepsilon}\left(x_{\varepsilon}\right)}{\varepsilon}}+o\left(\frac{1}{\varepsilon} e^{-\frac{\psi_{\varepsilon}\left(x_{\varepsilon}\right)}{\varepsilon}}\right), \quad$ in the Dirichlet case, or

$(5.15) \nabla K_{\varepsilon}\left(x_{\varepsilon}\right)=\frac{1}{\varepsilon} \gamma \alpha\left(x_{0}\right) e^{-\frac{\psi_{\varepsilon}\left(x_{\varepsilon}\right)}{\varepsilon}}+o\left(\frac{1}{\varepsilon} e^{-\frac{\psi_{\varepsilon}\left(x_{\varepsilon}\right)}{\varepsilon}}\right)$, in the Neumann case, where $\alpha\left(x_{0}\right) \in \partial \mathrm{d}_{\partial \Omega}\left(x_{0}\right)$ (see (3.1)) and $\gamma$ is a positive constant (see Proposition 5.2).

Proof. See Lemma (4.1) of [20]. 
THEOREM 5.7. Let $x_{\varepsilon}$ be a critical point of $K_{\varepsilon}$ such that $x_{0}=\lim _{\varepsilon \rightarrow 0} x_{\varepsilon} \in \Omega$. Then $x_{0}$ is a critical point of the distance function.

Proof. Since $x_{\varepsilon}$ is a critical point of $K_{\varepsilon}$ by Proposition (5.6) we get

$$
0=\nabla K_{\varepsilon}\left(x_{\varepsilon}\right)=\frac{1}{\varepsilon} \gamma \alpha\left(x_{0}\right) e^{-\frac{y_{\varepsilon}\left(x_{\varepsilon}\right)}{\varepsilon}}+o\left(\frac{1}{\varepsilon} e^{-\frac{\psi_{\varepsilon}\left(x_{\varepsilon}\right)}{\varepsilon}}\right),
$$

where $\alpha\left(x_{0}\right) \in \partial \mathrm{d}_{\partial \Omega}\left(x_{0}\right)$ and $\gamma$ is a positive constant. By (5.16) we deduce

$$
\gamma \alpha\left(x_{0}\right)+o(1)=0
$$

which implies $\alpha\left(x_{0}\right)=0$. Then $x_{0}$ is a critical point of the distance function since $0 \in \partial \mathrm{d}_{\partial \Omega}\left(x_{0}\right)$ (see Definition (1.7)).

Proof of Theorem (0.1). It follow by Proposition (5.2) and Theorem (5.5).

Proof of Theorem (0.2). It follow by Proposition (5.2) and Theorem (5.7).

6. Existence of multi-peak solutions. Let $\mathrm{H}_{\varepsilon}$ be the Hilbert space

$$
\mathrm{H}_{\varepsilon}=\left\{u \in \mathrm{H}^{2}\left(\Omega_{\varepsilon}\right) \mid \frac{\partial u}{\partial \nu_{\varepsilon}}=0 \text { on } \partial \Omega_{\varepsilon}\right\} \quad \text { in the Neumann case }
$$

Define

$$
\mathcal{S}_{\varepsilon}(u)=\Delta u-u+\left(u^{+}\right)^{p} \quad \text { for } \quad u \in \mathrm{H}_{\varepsilon} .
$$

Then solving equation (0.1) or equation (4.2) is equivalent to solve the following one

$$
\mathcal{S}_{\varepsilon}(u)=0, \quad u \in \mathrm{H}_{\varepsilon} .
$$

Fix $X=\left(x^{1}, \ldots, x^{K}\right) \in \mathcal{M}_{K}(\Omega)$. Let us consider the linearized operator $\mathcal{L}_{\varepsilon}$ : $\mathrm{H}_{\varepsilon} \longrightarrow \mathrm{L}^{2}\left(\Omega_{\varepsilon}\right)$ given by

$$
\mathcal{L}_{\varepsilon}(v)=\Delta v-v+p\left(\sum_{1}^{K} \mathcal{P}_{\Omega_{\varepsilon, x^{i}}} U\right)^{p-1} v
$$

It is easy to see that the cokernel of $\mathcal{L}_{\varepsilon}$ coincides with its kernel. Choose approximate cokernel and kernel as

$$
\begin{gathered}
\mathcal{K}_{\varepsilon, X}=\operatorname{span}\left\{\frac{\partial \mathcal{P}_{\Omega_{\varepsilon, x^{i}}} U}{\partial x_{j}^{i}} \mid i=1, \ldots, K, j=1, \ldots, N\right\} \subset \mathrm{H}_{\varepsilon}, \\
\mathcal{C}_{\varepsilon, X}=\operatorname{span}\left\{\frac{\partial \mathcal{P}_{\Omega_{\varepsilon, x^{i}}} U}{\partial x_{j}^{i}} \mid i=1, \ldots, K, j=1, \ldots, N\right\} \subset \mathrm{L}^{2}\left(\Omega_{\varepsilon}\right) .
\end{gathered}
$$

Now we state the following lemmas, which allow us to reduce problem (4.2) to a finite dimensional problem.

Lemma 6.1. For any compact set $C \subset \mathcal{M}_{K}(\Omega)$ there exists $\varepsilon_{0}>0$ such that for any $\varepsilon \in\left(0, \varepsilon_{0}\right)$ and $X \in C$ there exists a unique $\Phi_{\varepsilon, X} \in \mathcal{K}_{\varepsilon, X}^{\perp}$ such that

$$
\mathcal{S}_{\varepsilon}\left(\sum_{i=1}^{K} \mathcal{P}_{\Omega_{\varepsilon, x^{i}}} U+\Phi_{\varepsilon, X}\right) \in \mathcal{C}_{\varepsilon, X}
$$


Moreover $\Phi_{\varepsilon, X}$ is $C^{1}$ in $X$ and

$$
\left\|\Phi_{\varepsilon, X}\right\|_{\mathrm{H}^{2}\left(\Omega_{\varepsilon}\right)} \leq C e^{-(1+\sigma) \frac{\mathcal{D}_{K}(X)}{\varepsilon}},
$$

where $C$ is a positive constant, $\sigma=\min \{1, p-1\}$ and $\mathcal{D}_{K}$ is defined in (3.3).

Proof. The proof relies on a contraction mapping argument. The claim can be proved by collecting some results obtained in [9] and [23].

We now define the function $K_{\varepsilon}: \mathcal{M}_{K}(\Omega) \longrightarrow \mathbb{R}$ by

$$
K_{\varepsilon}\left(x^{1}, \ldots, x^{K}\right)=J_{\varepsilon}\left(\sum_{i=1}^{K} \mathcal{P}_{\Omega_{\varepsilon, x^{i}}} U+\Phi_{\varepsilon, X}\right),
$$

where the "rescaled" energy functional $J_{\varepsilon}: \mathrm{H}^{1}\left(\Omega_{\varepsilon}\right) \longrightarrow \mathbb{R}$ is defined in (5.3).

Firstly we compute the asymptotic expansion of $K_{\varepsilon}$.

Proposition 6.2. $X_{\varepsilon}=\left(x_{\varepsilon}^{1}, \ldots, x_{\varepsilon}^{K}\right)$ is a critical point of $K_{\varepsilon}$ if and only if $u_{\varepsilon}=\sum_{i=1}^{K} \mathcal{P}_{\Omega_{\varepsilon, x_{\varepsilon}^{i}}} U+\Phi_{\varepsilon, X_{\varepsilon}}$ is a solution of (4.2). Moreover the following estimate holds uniformly on compact sets of $\mathcal{M}_{K}(\Omega)$

$$
K_{\varepsilon}(x)=K A-\frac{1}{2} \gamma e^{-\frac{\Phi_{\varepsilon}(X)}{\varepsilon}}+o\left(e^{-\frac{\psi_{\varepsilon}(x)}{\varepsilon}}\right)
$$

where

$$
A=\frac{1}{2} \int_{\mathbb{R}^{N}}\left(|\nabla U|^{2}+U^{2}\right)-\frac{1}{p+1} \int_{\mathbb{R}^{N}} U^{p+1}, \gamma=\int_{\mathbb{R}^{N}} U^{p}(y) e^{-y_{1}} d y .
$$

Proof. See [9] and [23]).

The next results play a crucial role in connecting the topological structure of the sublevels of the function $\mathcal{D}_{K}$ with the topological structure of the sublevels of the function $K_{\varepsilon}$.

LeMma 6.3. Let $X_{1}^{\varepsilon}, X_{2}^{\varepsilon}$ be sequences in $\mathcal{M}_{K}(\Omega)$ such that $\lim _{\varepsilon \rightarrow 0} X_{1}^{\varepsilon}=X_{1} \in$ $\mathcal{M}_{K}(\Omega), \lim _{\varepsilon \rightarrow 0} X_{2}^{\varepsilon}=X_{2} \in \mathcal{M}_{K}(\Omega)$ and $\mathcal{D}_{K}\left(X_{1}\right)<\mathcal{D}_{K}\left(X_{2}\right)$. Then there exists $\varepsilon_{0}>0$ such that for any $\varepsilon \in\left(0, \varepsilon_{0}\right)$

$$
K_{\varepsilon}\left(X_{1}^{\varepsilon}\right)<K_{\varepsilon}\left(X_{2}^{\varepsilon}\right)
$$

Proof. We argue as in the proof of Lemma (5.3) using asymptotic expansion (6.3).

LeMma 6.4. Let $C_{1}, C_{2}$ be two compact subsets of $\mathcal{M}_{K}(\Omega)$. If

$$
\min _{X \in C_{1}} \mathcal{D}_{K}(X)>\max _{X \in C_{2}} \mathcal{D}_{K}(X)
$$

then there exists $\varepsilon_{0}>0$ such that for any $\varepsilon \in\left(0, \varepsilon_{0}\right)$

$$
\min _{X \in C_{1}} K_{\varepsilon}(X)>\max _{X \in C_{2}} K_{\varepsilon}(X)
$$


Now we prove that a suitable critical point of the function $\mathcal{D}_{K}$ generates a critical point of $K_{\varepsilon}$.

THEOREM 6.5. Let $c$ be a critical value topologically nontrivial of the function $\mathcal{D}_{K}$ (see Definition (2.4)). Then there exists a sequence $\left(X_{\varepsilon}\right)$ of critical points of $K_{\varepsilon}$ such that $\lim _{\varepsilon \rightarrow 0} X_{\varepsilon}=X_{0}, \mathcal{D}_{K}\left(X_{0}\right)=c$ and $X_{0} \in \mathcal{M}_{K}(\Omega)$.

Proof. By definition (2.4) there exist a family of $\partial Q_{\delta}, Q_{\delta}, A_{\delta}$ and $S_{\delta}$ of $\mathcal{M}_{K}(\Omega)$, which satisfy (2.1), (2.2), (2.3) and (2.6), namely:

$$
\max _{X \in \partial Q_{\delta}} \mathcal{D}_{K}(X)<\min _{X \in S_{\delta}} \mathcal{D}_{K}(X) \leq c \leq \max _{X \in Q_{\delta}} \mathcal{D}_{K}(X)<\min _{X \in A_{\delta}} \mathcal{D}_{K}(X)
$$

and

$$
\lim _{\delta \rightarrow 0} \min _{X \in S_{\delta}} \mathcal{D}_{K}(X)=\lim _{\delta \rightarrow 0} \max _{X \in Q_{\delta}} \mathcal{D}_{K}(X)=c .
$$

Then by (6.5) of Lemma (6.4) for any $\delta$ small enough there exists $\varepsilon_{0}(\delta)>0$ such that for any $\varepsilon \in\left(0, \varepsilon_{0}\right)$

$$
\max _{X \in \partial Q_{\delta}} K_{\varepsilon}(X)<\min _{X \in S_{\delta}} K_{\varepsilon}(X) \leq \max _{X \in Q_{\delta}} K_{\varepsilon}(X)<\min _{X \in A_{\delta}} K_{\varepsilon}(X) .
$$

Finally we want to show that a family of critical points of $K_{\varepsilon}$ converges to a critical point of the function $\mathcal{D}_{K}$. Firstly we have to compute the asymptotic expansion of the gradient of $K_{\varepsilon}$.

First of all we have to compute the expansion of the gradient of $K_{\varepsilon}$.

Proposition 6.6. For any $X \in \mathcal{M}_{K}(\Omega)$

$$
\nabla K_{\varepsilon}(X)=\frac{\gamma}{\varepsilon} \beta_{\varepsilon}(X) e^{-\frac{\Phi_{\varepsilon}(X)}{\varepsilon}}+o\left(e^{-\frac{\Phi_{\varepsilon}(X)}{\varepsilon}}\right),
$$

where $\beta_{\varepsilon}(X) \in \partial \mathcal{D}_{K}(X)$ (see Lemma sottodifffik)) and $\gamma$ is a positive constant (see Theorem (6.2)).

Proof. See Lemma (5.1) of [21].

THEOREM 6.7. Let $X_{\varepsilon}=\left(x_{\varepsilon}^{1}, \ldots, x_{\varepsilon}^{K}\right)$ be a critical point of $K_{\varepsilon}$ such that for $i=1, \ldots, K x_{0}^{i}=\lim _{\varepsilon \rightarrow 0} x_{\varepsilon}^{i} \in \Omega$. Then $X_{0}=\left(x_{0}^{1}, \ldots, x_{0}^{K}\right) \in \mathcal{M}_{K}(\Omega)$ and $X_{0}$ is a critical point of the function $\mathcal{D}_{K}$.

Proof. First of all we prove that $\left(x_{0}^{1}, \ldots, x_{0}^{K}\right) \in \mathcal{M}_{K}(\Omega)$, namely $x_{0}^{i} \neq x_{0}^{j}$ if $i \neq j$ (see [21], Theorem (6.1)).

Secondly we show that $X_{0}$ is a critical point of the function $\mathcal{D}_{K}$. Since $X_{\varepsilon}$ is a critical point of $K_{\varepsilon}$ by Proposition (6.6) we get

$$
0=\nabla K_{\varepsilon}\left(X_{\varepsilon}\right)=\frac{1}{\varepsilon} \gamma \beta\left(X_{\varepsilon}\right) e^{-\frac{\Phi_{\varepsilon}\left(X_{\varepsilon}\right)}{\varepsilon}}+o\left(\frac{1}{\varepsilon} e^{-\frac{\Phi_{\varepsilon}\left(X_{\varepsilon}\right)}{\varepsilon}}\right),
$$

where $\beta\left(X_{\varepsilon}\right) \in \partial \mathcal{D}_{K}\left(X_{\varepsilon}\right)$ and $\gamma$ is a positive constant. By (6.10) we deduce

$$
\beta\left(X_{\varepsilon}\right)+o(1)=0
$$

Let $X_{0}=\lim _{\varepsilon \rightarrow 0} X_{\varepsilon}$. By using Remark (1.4) we get $\lim _{\varepsilon \rightarrow 0} \beta\left(X_{\varepsilon}\right)=\beta\left(X_{0}\right) \in \partial \mathcal{D}_{K}\left(X_{0}\right)$ and by (6.11) we deduce that $\beta\left(X_{0}\right)=0$. Then $X_{0}$ is a critical point of the function $\mathcal{D}_{K}$ since $0 \in \partial \mathcal{D}_{K}\left(X_{0}\right)$ (see Definition (1.7)).

Proof of Theorem (0.3). It follow by Proposition (6.2) and Theorem (6.5).

Proof of Theorem (0.4). It follow by Proposition (6.2) and Theorem (6.7). 


\section{Examples.}

EXAMPLE 7.1. (A domain with one hole) Let $\Omega=\Sigma \backslash \bar{\sigma}$ where $\sigma \subset \Sigma$ are open sets. Assume $\max _{\Omega} \mathrm{d}_{\partial \Omega}>\frac{1}{2} \operatorname{dist}(\partial \sigma, \partial \Sigma)$. Then $c_{1}=\mathrm{d}_{\partial \Omega}\left(x_{1}\right)=\max _{\Omega} \mathrm{d}_{\partial \Omega}$ and $c_{2}=\mathrm{d}_{\partial \Omega}\left(x_{2}\right)=\frac{1}{2} \operatorname{dist}(\partial \sigma, \partial \Sigma)$ are two critical values topologically nontrivial of the distance function.

Proof. The existence of $c_{1}$ is trivial. Let us prove the existence of $c_{2}$. Let $y_{0} \in \partial \Sigma$ and $z_{0} \in \partial \sigma$ such that $\left|y_{0}-z_{0}\right|=\operatorname{dist}(\partial \sigma, \partial \Sigma)$. Set $x_{0}=\frac{y_{0}+z_{0}}{2}$. Then $\mathrm{d}_{\partial \Omega}\left(x_{0}\right)=$ $\frac{1}{2}\left|y_{0}-z_{0}\right|$. Let:

$$
S=\left\{x \in \Sigma \mid \operatorname{dist}(x, \partial \sigma)=\mathrm{d}_{\partial \Omega}\left(x_{0}\right)\right\}
$$

and

$$
Q=\left\{t y_{0}+(1-t) z_{0} \mid t \in[\delta, 1-\delta]\right\} \quad \text { for some } \delta>0
$$

Then it is easy to prove that the sets $Q$ and $S$ satisfies assumptions (2.1), (2.2) and (2.3):

$$
\max _{x \in \partial Q} \mathrm{~d}_{\partial \Omega}(x)<\min _{x \in S} \mathrm{~d}_{\partial \Omega}(x)=\mathrm{d}_{\partial \Omega}\left(x_{0}\right)=\max _{x \in Q} \mathrm{~d} \partial \Omega(x)
$$

That proves that $\mathrm{d}_{\partial \Omega}\left(x_{0}\right)$ is a critical value topologically nontrivial of the distance function in the sense of Definition (2.4).

EXAMPLE 7.2. (A domain with two holes) Let $\Omega=\Sigma \backslash\left(\bar{\sigma}_{1} \cup \bar{\sigma}_{2}\right)$ where $\sigma_{i} \subset \Sigma$ are open sets, $\sigma_{1}$ and $\sigma_{2}$ are strictly convex and $\sigma_{1} \cap \sigma_{2}=\emptyset$. Assume

$$
\operatorname{dist}\left(\partial \sigma_{1}, \partial \Sigma\right)<\operatorname{dist}\left(\partial \sigma_{2}, \partial \Sigma\right)<\operatorname{dist}\left(\partial \sigma_{1}, \partial \sigma_{2}\right)
$$

Then $c_{1}=\mathrm{d}_{\partial \Omega}\left(x_{1}\right)=\max _{\Omega} \mathrm{d}_{\partial \Omega}, c_{2}=\mathrm{d}_{\partial \Omega}\left(x_{2}\right)=\frac{1}{2} \operatorname{dist}\left(\partial \sigma_{1}, \partial \Sigma\right), c_{3}=\mathrm{d}_{\partial \Omega}\left(x_{3}\right)=$ $\frac{1}{2} \operatorname{dist}\left(\partial \sigma_{2}, \partial \Sigma\right)$ and $c_{4}=\mathrm{d}_{\partial \Omega}\left(x_{4}\right)=\frac{1}{2}$ dist $\left(\partial \sigma_{1}, \partial \sigma_{2}\right)$ are four critical values topologically nontrivial of the distance function.

Proof. The existence of $c_{1}$ is trivial. First of all we prove the existence of $c_{2}$ and $c_{3}$. Let $i=1,2$. Let $y_{0}^{i} \in \partial \Sigma$ and $z_{0}^{i} \in \partial \sigma_{i}$ such that $\left|y_{0}^{i}-z_{0}^{i}\right|=\operatorname{dist}\left(\partial \sigma_{i}, \partial \Sigma\right)$. Set $x_{0}^{i}=\frac{y_{0}^{i}+z_{0}^{i}}{2}$. Then $\mathrm{d}_{\partial \Omega}\left(x_{0}^{i}\right)=\frac{1}{2}\left|y_{0}^{i}-z_{0}^{i}\right|$. Let:

$$
S_{i}=\left\{x \in \Sigma \mid \operatorname{dist}\left(x, \partial \sigma_{i}\right)=\mathrm{d}_{\partial \Omega}\left(x_{0}^{i}\right)\right\}
$$

and

$$
Q_{i}=\left\{t y_{0}^{i}+(1-t) z_{0}^{i} \mid t \in[\delta, 1-\delta]\right\} \quad \text { for some } \delta>0 \text {. }
$$

We point out that (7.1) ensures that $\mathrm{d}_{\partial \Omega}(x)=\operatorname{dist}\left(x, \partial \sigma_{i}\right) \quad \forall x \in S_{i}$. Then it is easy to prove that the sets $Q_{i}$ and $S_{i}$ satisfies assumptions (2.1), (2.2) and (2.3):

$$
\max _{x \in \partial Q_{i}} \mathrm{~d}_{\partial \Omega}(x)<\min _{x \in S_{i}} \mathrm{~d}_{\partial \Omega}(x)=\mathrm{d}_{\partial \Omega}\left(x_{0}^{i}\right)=\max _{x \in Q_{i}} \mathrm{~d}_{\partial \Omega}(x) .
$$

That proves that $\mathrm{d}_{\partial \Omega}\left(x_{0}^{i}\right)$ is a critical value topologically nontrivial of the distance function in the sense of Definition (2.4). 
Now we prove the existence of $c_{4}$. Since $\sigma_{1}$ and $\sigma_{2}$ are strictly convex there exist exactly two points $z_{1} \in \partial \sigma_{1}$ and $z_{2} \in \partial \sigma_{2}$ such that $\left|z_{1}-z_{2}\right|=\operatorname{dist}\left(\partial \sigma_{1}, \partial \sigma_{2}\right)$. Set $x_{0}=\frac{z_{1}+z_{2}}{2}$. Then $\mathrm{d}_{\partial \Omega}\left(x_{0}\right)=\frac{1}{2}\left|z_{1}-z_{2}\right|$. Let for some $\delta>0$

$$
Q=\left\{t z_{1}+(1-t) z_{2} \mid t \in[\delta, 1-\delta]\right\}
$$

$S=\left\{\right.$ hyperplane perpendicular to $Q$ crossing the point $\left.x_{0}\right\} \cap B\left(x_{0}, \delta\right)$.

and

$A=\left\{\right.$ hyperplane perpendicular to $Q$ crossing the point $\left.x_{0}\right\} \cap \partial B\left(x_{0}, \delta\right)$.

Then it is easy to prove that the sets $Q, S$ and $A$ satisfies assumptions (2.1), (2.2) and (2.3):

$$
\max _{x \in \partial Q} \mathrm{~d}_{\partial \Omega}(x)<\min _{x \in S} \mathrm{~d}_{\partial \Omega}(x)=\mathrm{d}_{\partial \Omega}\left(x_{0}\right)=\max _{x \in Q} \mathrm{~d}_{\partial \Omega}(x)<\min _{x \in A} \mathrm{~d}_{\partial \Omega}(x) .
$$

That proves that $\mathrm{d}_{\partial \Omega}\left(x_{0}\right)$ is a critical value topologically nontrivial of the distance function in the sense of Definition (2.4).

If the domain has a lot of holes the existence of many critical values topologically nontrivial of the distance function strongly depends on the geometry of the holes.

EXAMPLE 7.3. (A domain with $k$ handles) Let $\Omega$ be a domain with $k$ handles. Then there exist at least $2 k+1$ distinct critical values topologically nontrivial of the distance function: $k+1$ local maxima of $\mathrm{d}_{\partial \Omega}$ and $k$ local saddle levels.

Note that we can have more than a critical point at the same level.

EXAMPLE 7.4. Let $\Omega$ be the dumbell. Then $d$ is a critical value topologically non trivial of $\mathcal{D}_{2}$. Moreover one can choose the dumbell so that $(0, d, 0,-d)$ is the unique critical point of $\mathcal{D}_{2}$ at level d. and set

We prove that the point $(0, d, 0,-d)$ is a "local saddle point" of $\mathcal{D}_{2}$. Fix $\varepsilon>0$

$$
\begin{aligned}
Q_{\varepsilon}= & \left\{\left(0, x_{2}^{1}\right) \in \Omega \|\left|x_{2}^{1}-d\right| \leq \varepsilon\right\} \times\left\{\left(0, x_{2}^{2}\right) \in \Omega \|\left|x_{2}^{2}+d\right| \leq \varepsilon\right\} \\
\partial Q_{\varepsilon}= & \left(\{(0, d \pm \varepsilon)\} \times\left\{\left(0, x_{2}^{2}\right) \in \Omega \|\left|x_{2}^{2}+d\right| \leq \varepsilon\right\}\right) \\
& \cup\left\{\left(0, x_{2}^{1}\right) \in \Omega \|\left|x_{2}^{1}-d\right| \leq \varepsilon\right\} \times\{(0,-d \pm \varepsilon)\}
\end{aligned}
$$

For $\delta>0$ and $\rho>0$ set

$$
\begin{aligned}
C_{\delta}= & \left\{x \in \Omega \mid \mathrm{d}_{\partial \Omega}(x)=d+\delta\right\} \\
S_{\delta}= & \left(B((0, d), \rho) \cap C_{\delta}\right) \times\left(B((0,-d), \rho) \cap C_{\delta}\right) \\
A_{\delta}= & \left(\partial B((0, d), \rho) \cap C_{\delta}\right) \times\left(B((0,-d), \rho) \cap C_{\delta}\right) \\
& \cup\left(B((0, d), \rho) \cap C_{\delta}\right) \times\left(\partial B((0,-d), \rho) \cap C_{\delta}\right) .
\end{aligned}
$$

Then by using Remark (2.2) it is easy to check that if we choose $\delta$ and $\rho$ small enough $\partial Q_{\delta}, Q_{\delta}, A_{\delta}$ and $S_{\delta}$ are subsets of $\Omega \times \Omega$ which satisfy (2.1), (2.2) and (2.3) and

$$
\max _{\partial Q_{\delta}} \mathcal{D}_{2}=d-\varepsilon<\min _{S_{\delta}} \mathcal{D}_{2}=d-\delta<d=\max _{Q_{\delta}} \mathcal{D}_{2}<\min _{A_{\delta}} \mathcal{D}_{2}=d+\delta .
$$


Moreover $\lim _{\delta \rightarrow 0} \min _{S_{\delta}} \mathcal{D}_{2}=d$. By Lemma (3.7) we deduce that the sets $\{X \in$ $\mathcal{M}_{K}(\Omega)$ s.t. $\left.c \leq \mathcal{D}_{K}(X)\right\}$ are complete for any $c>0$. Therefore $d$ is a critical value of $\mathcal{D}_{2}$.

Finally by using Remark (3.4) and Remark (3.3) one can construct a dumbell in such a way the distance function is differentiable at any $x$ with $\mathrm{d}_{\partial \Omega}(x)=d$ and by using Corollary (3.9) one can check that $(0, d, 0,-d)$ is the unique critical point of $\mathcal{D}_{2}$ at level $d$.

REMARK 7.5. We note that in the dumbell the points $(a, r, a,-r)$ and $(b, R, b,-R)$ are two local maximum points of the function $\mathcal{D}_{2}$ at different levels $\mathcal{D}_{2}(a, r, a,-r)=r$ and $\mathcal{D}_{2}(b, R, b,-R)=R$.

However we point out that such points are not isolated critical points of $\mathcal{D}_{2}$ at levels $r$ and $R$, respectively. In fact if $x^{1}$ is a point close enough to the point $(a, r)$, which belongs to the sphere centered at $(a, 0)$ with radius $r$ and $x^{2}$ is the point diametrically opposite, it is easy to check that $\left(x^{1}, x^{2}\right)$ is a local maximum point of the function $\mathcal{D}_{2}$ at level $r$.

\section{REFERENCES}

[1] A. BAHRI, Critical point at infinity in some variational problems, Pitman Research Notes Math., 182 Longman House, Harlow (1989).

[2] V. BencI, G. CERAMI, The effect of the domain topology on the number of positive solutions of nonlinear elliptic problem, Arch. Rat. Mech. Anal., 114 (1991), pp. 79-93.

[3] V. Benci, G. Cerami, D. PAssaseo, On the number of the positive solutions of some nonlinear elliptic problems, Nonlinear Analysis: A Tribute in Honour of Giovanni Prodi, S.N.S. (1991), pp. 93-107.

[4] A. BAHRI, Y.Y.LI, O.REY, On a variational problem with lack of compactness: the topological effect of the critical points at infinity, Calculus of Variat., 3 (1995), pp. 67-93.

[5] P.W.BAtes, G.Fusco, Equilibria with many nuclei for the Cahn-Hilliard equation, J. Differential Equations, 160 (2000), pp. 283-356.

[6] H. Berestycki, P.L. Lions, Nonlinear scalar field equations $I$ : existence of a ground state, Arch. Rat. Mech. Anal., 82 (1983), pp. 313-346.

[7] D.CAO, E.N.DANCER, E.NoussaIR, S.YAN, On the existence and profile of multi-peaked solutions to singularly perturbed semilinear Dirichlet problems, Disc. Cont. Dyn. Syst., 2 (1996), pp. 221-236.

[8] D.CAO,E.NouSSAIR, Multi-peak solutions for a singularly perturbed semilinear elliptic problem, preprint.

[9] G. CERAMI, J. WEI, Multplicity of multiple interior peaks solutions for some singularly perturbed Neumann problems, Intern. Math. Res. Notes, 12 (1998), pp. 601-626.

[10] K.C. Chang, Variational methods for non-differentiable functionals and their applications to partial differential equations, J. Math. Anal. Appl., 80 (1981), pp. 102-129.

[11] F.H. ClaRKE, Optimization and nonsmooth analysis, John Wiley and Sons (1983).

[12] E.N.DANCER, S.YAN, Multipeak solutions for a singularly perturbed Neumann problem, Pacific Journal Math., to appear.

[13] E.N.DANCER, S.YAN, A singularly perturbed elliptic problem in bounded domains with nontrivial topology, Adv. Diff. Equat., 4 (1999), pp. 347-368.

[14] E.N.DANCER, S.YAN, Effect of the domain geometry on the existence of multipeak solutions for an elliptic problem, Topol. Meth. Nonlin. Anal., to appear.

[15] E.N.DANCER, J.WEI, On the effect of domain topology in a singular perturbation problem, Topol. Meth. Nonlin. Anal., 11 (1998), pp. 227-248.

[16] M. DeL PINo, P. L. Felmer, J. WEI, On the role of distance function in some singular perturbation problems, Comm. in P.D.E., 25 (2000), pp. 155-177.

[17] M. Del PINo, P. L. FElmer, J. WEI, Multiple-peak solutions for some singular perturbation problems, submitted.

[18] A.Floer, A.Weinstein, Nonspreading wave packets for the cubic Scröedinger equation with a bounded potential, J. Funct. Anal., 69 (1986), pp. 397-408. 
[19] B. GidAs, W. N. NI, L. NiRENBERG, Symmetry of positive solutions of nonlinear elliptic equations in $\mathbb{R}^{N}$, Mathematical analysis and applications, Part A, Adv. Math. Suppl. Studies, 7A, Acad. Press, New York, 1981.

[20] M.Grossi, A.PISTOIA, On the effect of critical points of distance function in superlinear elliptic problems, Advances in Diff. Equat., to appear.

[21] M.Grossi, A.PISTOIA, J.WEI, Multipeak solutions for a semilinear Neumann problem via nonsmooth analysis, Calculus of Variat, to appear.

[22] C.GuI, Multipeak solutions for a semilinear Neumann problem, Duke Math. J., 84 (1996), pp. 739-769.

[23] C.GUI, J. WEI, Multiple interior peak solutions for a for some singularly perturbed Neumann problem, Journ. Diff. Equat., to appear.

[24] C.GUI, J. WEI, M.WINTER, Multiple boundary peak solutions for some singularly perturbed Neumann problems, Ann. Inst. H.Poincarè, to appear.

[25] M.KowALCZYK, Multiple spike layers in the shadow Gierer-Meinhardt system: existence of equilibria and quasi invariant manifold, Duke Math. J., 98 (1999), pp. 59-111.

[26] M. K. Kwong, Uniqueness of positive solutions of $\Delta u-u+u^{p}=0$ in $\mathbb{R}^{n}$, Arch. Rat. Mech. Anal., 105 (1989), pp. 243-266.

[27] Y.Y. LI, On a singularly perturbed equation with Neumann boundary condition, Comm. P.D.E., 23 (1998), pp. 487-545.

[28] Y.Y. Li, L. NIRENBERG, The Dirichlet problem for singularly perturbed elliptic equation, Comm. Pure Appl. Math., 51 (1998), pp. 1445-1490.

[29] C. LIN, W.M. NI, I. TAKAGI, Large amplitude stationary solutions to a chemotaxis system, J. Diff. Eqns., 72 (1988), pp. 1-27.

[30] A. Marino, A.M. Micheletti, A. Pistoia, Some variational results on semilinear problems with asymptotically nonsimmetric behaviour, Nonlinear Analysis "A Tribute in honor of G.Prodi, S.N.S. Pisa (1991), pp. 243-256.

[31] W.M. NI, I. TAKAGI, On the shape of least energy solutions to a semilinear neumann problem, Comm. Pure Math. Appl., 41 (1991), pp. 819-851.

[32] W.M. NI, I. TAKAGI, Locating the peaks of least energy solutions to a semilinear Neumann problem, Duke Math. Journ., 70 (1992), pp. 247-281.

[33] W.M. NI, I. TAKAGI, Point-condensation generated by a reaction-diffusion system in axially symmetric domains, Japan J.Industr.Appl. Math., 12 (1995), pp. 327-365.

[34] W.M. NI, J. WEI, On the location and profile of spike-layer solutions to singularly perturbed semilinear Dirichlet problems, Comm. Pure Math. Appl., 48 (1995), pp. 731-768.

[35] A.PISTOIA, On the existence of symmetric solutions to some singularly perturbed semilinear elliptic problems, Asympt. Anal., 22 (2000), pp. 149-161.

[36] M. Ramos, Teoremas de enlace na teoria dos pontos criticos, Textos de Matematica, Dep. Matematica, Univ. de Lisboa, (1993).

[37] S.YAN, On the number of interior multipeak solutions for singularly perturbed Neumann problems, preprint.

[38] Z.Q. WANG, On the existence of multiple single-peaked solution for a semilinear Neumann problem, Arch. Rat. Mech. Anal., 120 (1992), pp. 375-399.

[39] J. WEI, On the boundary spike layer solutions to a singularly perturbed Neumann problem, J. Diff. Equat., 134 (1997), pp. 104-133.

[40] J. WEI, On the interior spike solutions for some singular perturbation problems, Proc. Roy. Soc. Edinb., 128 (1998), pp. 849-874.

- [41] J. WEI, On the construction of interior spike layer solutions to a singularly perturbed semilinear Neumann problem, Tohoku Math. J., 50 (1998), pp. 159-178.

[42] J. WEI, On the construction of interior spike layer solutions to a singularly perturbed semilinear Neumann problem, Partial Differential Equations: Theory and Numerical solution, CRC Press, (1998), pp. 336-349.

[43] J. WEI, Conditions for two-peaked solutions of a singularly perturbed elliptic equation, Manuscript. Math., 96 (1998), pp. 113-131.

[44] J. WEI, M.WINTER, Multiple boundary spike solutions for a wide class of singular perturbation problems, J. London Math. Soc., 59 (1999), pp. 82-101. 
A. PISTOIA 